\title{
MÉTODOS PARA DETERMINAR LAS CASAS DEL HORÓSCOPO EN LA ASTROLOGÍA MEDIEVAL ÁRABE ${ }^{1}$
}

\author{
METHODS FOR DETERMINING THE HOUSES OF THE \\ HOROSCOPES IN MEDIEVAL ARABIC ASTROLOGY
}

\author{
Josep CASULLERAS \\ Universitat de Barcelona \\ To the memory of \\ Professor J.D. North \\ (May 19, 1934-October 31, 2008)
}

\begin{abstract}
Este trabajo repasa los distintos métodos de cálculo que los astrónomos y matemáticos árabes medievales desarrollaron para ser aplicados a la práctica astrológica de la división de casas. Partiendo de una clasificación de estos métodos establecida por J.D. North (1986) y ampliada por E.S. Kennedy (1996), se recoge la información que se halla en estudios anteriores y se presentan nuevos datos como resultado del análisis de fuentes exploradas más recientemente, destacando ciertos elementos pertenecientes a una tradición astrológica occcidental.
\end{abstract}

Palabras clave: al-Andalus; astrología; división de casas; matemática aplicada; trigonometría esférica.
This paper revises the different methods of computation devised by Medieval Arabic astronomers and mathematicians for the astrological practice of the division of houses. Starting from a classification of these methods established by J.D. North (1986) and extended by E.S. Kennedy (1996), I summarize the available information contained in previous studies and present new data emerging from the analysis of more recently explored sources, emphasizing certain features of a Western astrological tradition.

Key words: al-Andalus; Applied mathematics; Astrology; Division of houses; Spherical trigonometry.

\section{Introducción}

En las últimas décadas, han merecido ser objeto de interés por parte de los historiadores de la astronomía y la matemática árabes los procedimientos de cálculo y los planteamientos geométricos que se aplican en el mundo árabe medieval a la resolución de tres cuestiones

${ }^{1}$ Este trabajo se ha finalizado en el marco del programa de investigación "La evolución de la ciencia en la sociedad de al-Andalus desde la Alta Edad Media al pre-Renacimiento", cofinanciado por el Ministerio de Ciencia e Innovación (FFI 2008-00234 FILO) y el Fondo Europeo de Desarrollo Regional (FEDER). 
fundamentales para la práctica de la astrología natalicia, provenientes de la tradición griega: la división de casas (taswiyat al-buyūt), los aspectos planetarios o proyección de rayos (mațaḥ al-šu ' $\bar{a}$ 'át) y el sistema de progresiones (tasyir $)^{2}$. De este modo, la práctica de la astrología, en tanto que necesita disponer de definiciones geométricas y operaciones de cálculo específicas y distintas de las que se aplican a la astronomía, se ha revelado como uno de los campos que integran el ámbito general de la matemática aplicada. Diversos trabajos han contribuido a identificar los métodos diseñados por los astrónomos matemáticos para uso de los astrólogos practicantes, estableciendo clasificaciones para estos procedimientos que permiten disponer de una nomenclatura útil y de unas definiciones concretas a la hora de analizar fuentes relacionadas con la astrología ${ }^{3}$. En este sentido, el presen-

2 Sobre estos conceptos en la tradición griega puede consultarse, por ejemplo, el clásico Bouché-Leclerq, A., L'astrologie grecque, París, 1899 (reimp. Bruselas, 1963), 279 (casas), 280-285 (aspectos), 411-422 (progresiones).

${ }^{3}$ Como punto de partida para el estudio de materiales astrológicos conservados en fuentes antiguas y medievales, pueden considerarse los siguientes trabajos, enumerados por orden de aparición, y las referencias que contienen: Kennedy, E.S. y Krikorian, H., "The Astrological Doctrine of Projecting the Rays", Al-Abhath, 25 (1972), 3-15 (reimp. Kennedy, E.S., "Colleagues and former students", en Studies in the Islamic Exact Sciences, Beirut, 1983, 372-384); North, J.D., Horoscopes and History, Londres, 1986; Hogendijk, J.P. "The Mathematical Structure of Two Islamic Astrological Tables for "Casting the Rays"', Centaurus, 32 (1989), 171-202; Kennedy, E.S., "Ibn Mu'ādh on the Astrological Houses", Zeitschrift für Geschichte der Arabish-Islamischen Wissenschaften, 9 (1994), 153-160; idem, "The Astrological Houses as Defined by Medieval Islamic Astronomers", en J. Casulleras y J. Samsó (eds.), From Baghdad to Barcelona. Studies in the Islamic Exact Sciences in Honour of Prof. Juan Vernet, Barcelona, 1996, II, 535-578 (ambos reimp. en Kennedy, E.S., Astronomy and Astrology in Medieval Islamic World, Aldershot, Variorum, 1998, n. ${ }^{\circ}$ XVI y XIX); North, J.D., "A reply to Prof. E.S. Kennedy”, en J. Casulleras y J. Samsó (eds.), From Baghdad to Barcelona, 579-582; Calvo, E., "La résolution graphique des questions astrologiques à al-Andalus", Histoire des Mathematiques Arabes: Actes du $3^{\text {me }}$ Colloque Maghrébin sur l'Histoire des Mathématiques Arabes, Tipaza, Alger, Algérie, 1-3 Décembre 1990, Alger, 1998, 31-44; Hogendijk, J.P., "Progressions, Rays and Houses in Medieval Islamic Astrology: A Mathematical Classification”, artículo inédito del que dispongo en forma de fotocopia gracias a la generosidad de su autor, presentado en la Dibner Institute Conference, New Perspectives on Science in Medieval Islam, Cambridge, Mass., 6-8 noviembre 1998; Samsó, J. y Berrani, H., "World astrology in eleventh-century al-Andalus: the Epistle on Tasyir and the Projection of Rays by al-Istiji”", Journal of Islamic Studies, 10, 3 (1999), 293-312; Casulleras, J., "Ibn Mu'ādh on the Astrological Rays", Suhayl, 4 (2004), 385-402; Hogendijk, J.P., "Applied Mathematics in Eleventh century al-Andalus: Ibn Mu'ādh al-Jayyānī and his computation of astrological houses and aspects", Centaurus, 47 (2005), 87-114; Samsó, J. y Berrani, H., "The Epistle on Tasyīr and the projection of rays by Abū Marwān al-Istijī”, Suhayl, 5 (2005), 163-242; Casulleras, J., "El cálculo de aspectos o la proyección de rayos en la astrología medieval árabe", Archives

Al-Qanțara (AQ) XXX 1, enero-junio 2009, pp. 41-67 ISSN 0211-3589 
te artículo ofrece una síntesis de los procedimientos destinados a la primera de las tres prácticas mencionadas, la división de casas, teniendo en cuenta algunas fuentes de información hasta hace poco prácticamente desconocidas o ignoradas desde este punto de vista.

\section{Símbolos utilizados}

A continuación se describen los símbolos que se usan en las páginas siguientes y que, en general, pueden representar un valor numérico, un arco o un punto de la esfera celeste, o bien una función cuando tienen argumentos entre paréntesis.

Figura 1

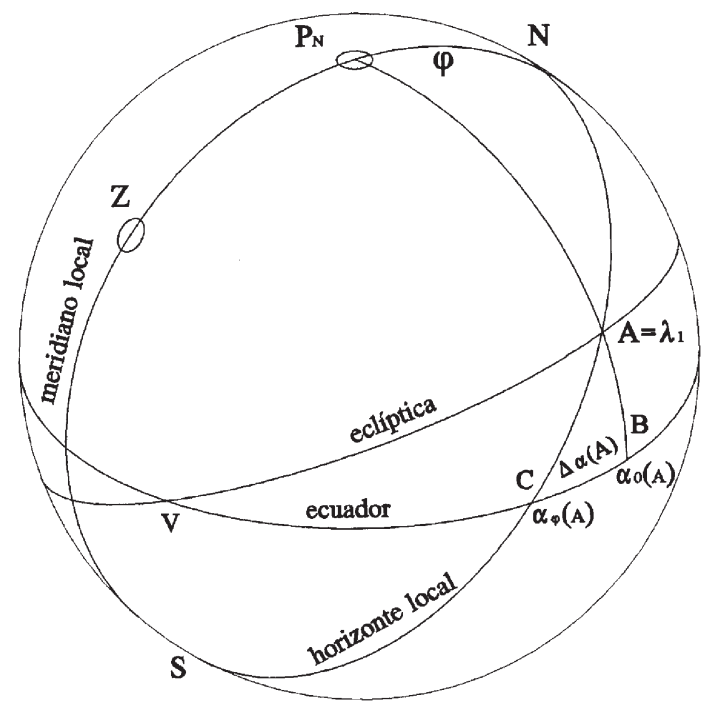

$\alpha_{0}$ ascensión recta. Arco del ecuador celeste comprendido entre el punto vernal (intersección del ecuador celeste con la eclíptica en Aries $0^{\circ}$ ) y el meridiano que pasa por un punto de la esfera celeste dado. Se mide en sentido inverso al de las agujas del reloj, es decir,

Internationales d'Histoire des Sciences, 57, 158 (2007), 25-46; idem, "Ibn 'Azzūz al-Qusantīnī's Tables for computing planetary aspects”, Suhayl, 7 (2007), 47-114. 
contrario al del movimiento diurno. En la figura 1, el arco $V B$ corresponde a la ascensión recta del grado ascendente, $\alpha_{0}(A)$.

$\alpha_{0}{ }^{-1} \quad$ función inversa de $\alpha 0$, devuelve como resultado la longitud eclíptica $\lambda$ correspondiente al grado del ecuador que representa $\alpha_{0}$.

$\alpha_{\varphi}$ ascensión oblicua. Arco del ecuador celeste que asciende simultáneamente con un arco de la eclíptica dado en una localidad de latitud $\varphi$. Su origen es el punto vernal y se cuenta en sentido inverso al del movimiento diurno. En la figura 1 se representa la ascensión oblicua del grado ascendente: $\alpha_{\varphi}(A)$, arco $V C$, para un horizonte de latitud $\varphi=P_{N} N$.

$\alpha_{\varphi}^{-1} \quad$ función inversa de $\alpha_{\varphi}$, devuelve como resultado la longitud eclíptica $\lambda$ correspondiente al grado del ecuador que representa $\alpha_{\varphi}$.

\section{Figura 2}

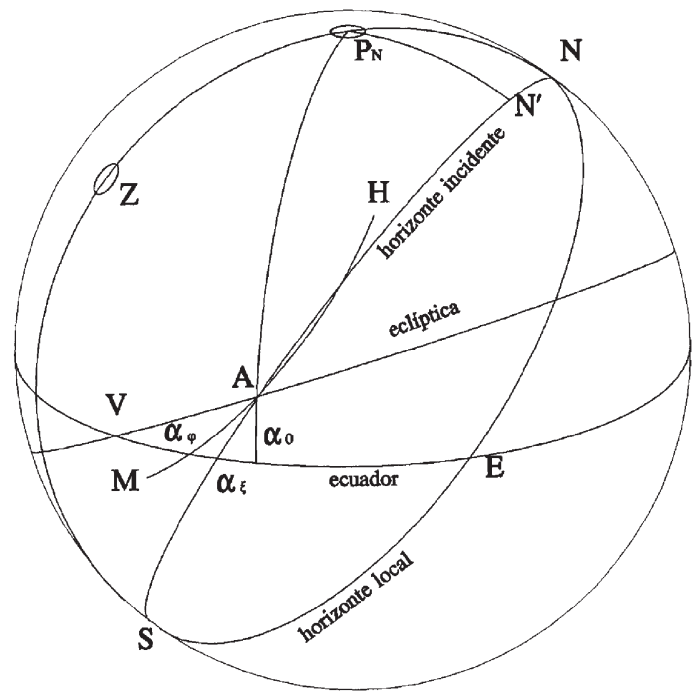

$\alpha_{\xi} \quad$ ascensión oblicua en un horizonte incidente de latitud $\xi$, siendo $\varphi>\xi>0^{\circ}$ (véase $\S 3$ ). El arco $M A H$ de la figura 2 representa la posición del horizonte local (SEN) cuando $A$ se halle sobre este círculo, y en ella se representan, para un grado de la eclíptica $A$, las tres proyecciones sobre el ecuador celeste correspondientes a su ascensión 
recta $\alpha_{0}$, a su ascensión oblicua $\alpha_{\varphi}$ para un horizonte de latitud $\varphi=P_{N} N$ y a su ascensión oblicua $\alpha \xi$ en el horizonte incidente que pasa por $A$, círculo $N N^{\prime} A S$, de latitud $\xi=P_{N} N^{\prime}$.

$\Delta_{\alpha}$ diferencia ascensional. También denominada ecuación del día y ecuación o exceso del arco semidiurno (ta 'dìl niṣf qaws al-nahār, faḍl niṣf al-nahār o nișf al-fậla). Para un grado $g$ de la eclíptica, $\Delta_{\alpha}(g)$ es la diferencia mínima entre su ascensión recta, $\alpha_{0}(g)$, y su ascensión oblicua, $\alpha_{\varphi}(g)$. En la figura 1, el arco del ecuador celeste $C B$ representa la diferencia ascensional del grado ascendente, $\Delta_{\alpha}(A)$.

$\varphi$ latitud geográfica. Distancia angular mínima, medida desde el centro de la tierra, entre la posición del observador y el ecuador terrestre. Positiva en el hemisferio norte y negativa en el hemisferio sur. Esta magnitud es equivalente a la altura del polo celeste norte sobre el horizonte del observador. Arco $P_{N} N$ en la figura 1.

$\xi \quad$ latitud geográfica distinta de $\varphi$ y comprendida entre $0^{\circ}$ y $\varphi$. Corresponde a la latitud de un supuesto horizonte incidente (véase $\S 3$ ). Arco $P N^{\prime}$ en la figura 3 .

Figura 3

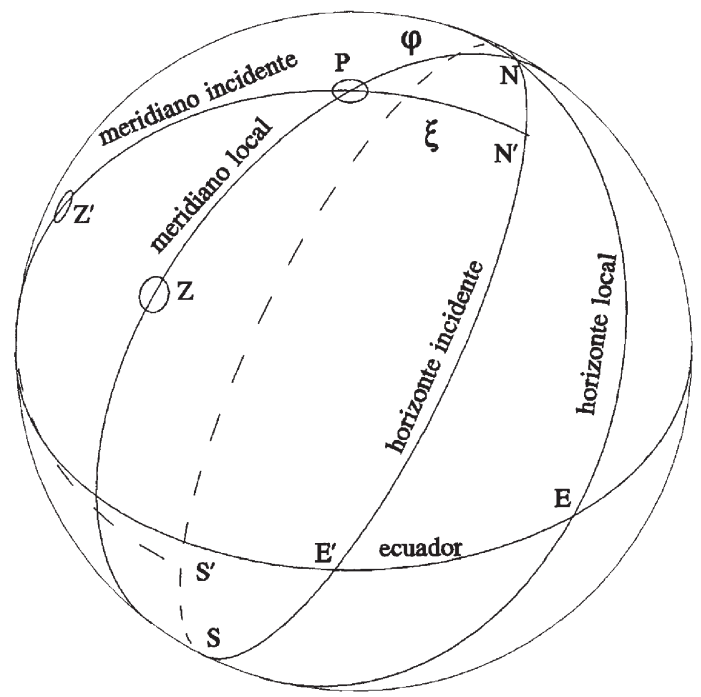

Al-Qantara (AQ) XXX 1, enero-junio 2009, pp. 41-67 ISSN 0211-3589 
$\lambda$ grado de longitud eclíptica. Contado desde Aries $0^{\circ}$ en sentido directo, contrario al del movimiento diurno. En la figura 1, el arco $V A$ corresponde a la longitud eclíptica del punto $A$.

$\lambda \mathrm{i}$ para $i=1 \ldots 12$, longitud eclíptica del principio de la casa astrológica $i$. En la figura 1, el mencionado arco $V A$ corresponde igualmente a la longitud del ascendente, $\lambda_{1}$.

I ... XII cifras romanas que indican casas astrológicas, se refieren a éstas en un sentido no restringido a su longitud eclíptica.

\section{Horizontes incidentes o círculos de posición}

El planteamiento geométrico de una buena parte de los métodos desarrollados para abordar tanto el problema de la división de casas como las cuestiones de los aspectos planetarios y las progresiones implica el uso de los denominados círculos de posición. Por este motivo, es útil anticipar en primer lugar este concepto que, aunque definible en términos astronómicos, se utiliza exclusivamente en fuentes astrológicas.

En la esfera celeste se definen los círculos de posición como los círculos máximos que intersecan al horizonte del observador en sus puntos Norte y Sur. El tercer punto de la esfera que define uno de estos círculos es alguno de los elementos significativos utilizados en la práctica astrológica. De modo análogo a la determinación de $\alpha_{\varphi}$ mediante el horizonte local, mediante un arco de un círculo de posición se determina la proyección de la posición de este objeto, o de su longitud eclíptica, sobre otro círculo máximo, frecuentemente el ecuador celeste $\left(\alpha_{\xi}\right)$. En algunas fuentes el círculo de posición se usa sin una denominación específica ${ }^{4}$, mientras que en otras se denomina horizonte incidente

${ }^{4}$ Éste es el caso, por ejemplo de la Risāla fì matrah al-šu 'ā ‘āt de Ibn Mu'ād̄ de Jaén (m. 1093), quien usa círculos correspondientes a esta definición en los algoritmos que desarrolla tanto para la división de casas como para la proyección de rayos. Sobre este autor, véanse Calvo, E. y Casulleras, J., "Ibn Mu'ād al-Ŷayyānī”, en J. Lirola (ed.), Enciclopedia de la Cultura Andalusí, IV, 2006, 197-201, n. ${ }^{\circ}$ 819; Calvo, E., "Ibn Mu'ādh al-Jayyānī, Abū 'Abd Allāh Muhammad", en T. Hockey (ed.), The Biographical Encyclopedia of Astronomers, New York, Springer, 2007 (versión en línea en http://islamsci.mcgill.ca/RASI/BEA/), 562-563. Respecto a su Risāla fì matrah al-šu 'à 'àt, véanse Kennedy, "Ibn Mu'ādh”; Hogendijk, "Applied Mathematics"; Casulleras, "Ibn Mu'ādh". Una primitiva definición de los círculos de posición se halla en el tratado de construcción del astrolabio de al-Fargānī (probablemente ca. 856-857), cf. al-Farghānī, On the Astrolabe. Arabic Text and Commentary by Richard Lorch, Wiesbaden, 2005, 5, 10, 60-63; agradezco esta información a Julio Samsó.

Al-Qanțara (AQ) XXX 1, enero-junio 2009, pp. 41-67 ISSN 0211-3589 
(ufuq hāadit $)^{5}$, puesto que equivale al horizonte de latitud $\xi$, comprendida entre $0^{\circ}$ y la latitud del observador, $\varphi$, por el que asciende o desciende (en este caso se usará $-\xi$ ) en ese momento el objeto en cuestión. En la figura 3, el círculo $N N^{\prime} E$ 'SS' representa uno de estos horizontes. Considerando que entre su punto Este $\left(E^{\prime}\right)$ y su punto Norte $\left(N^{\prime}\right)$ deben mediar $90^{\circ}$, la medida del arco $P N^{\prime}$, perteneciente al meridiano local de ese horizonte, nos da su latitud geográfica $\xi$.

\section{Métodos para la división de casas}

Una actividad fundamental de la astrología, el levantamiento del horóscopo, consiste en la representación gráfica de la esfera celeste en un momento determinado y en relación a un horizonte dado ${ }^{6}$. Una vez determinadas las longitudes eclípticas $(\lambda)$ de los elementos significativos para la práctica astrológica - esto es, principalmente, el Sol, la Luna, los nodos lunares (intersecciones de la órbita lunar con la eclíptica) y los planetas - ya sea por medio de tablas, reglas de cómputo aproximadas, efemérides, almanaques o instrumentos analógicos, la relación entre estas posiciones y el horizonte del lugar para el que se levanta el horóscopo se efectúa mediante el llamado sistema de casas celestes. Estas casas son, en general, doce divisiones formadas por doce semiplanos con una arista común que forman otros tantos diedros en la esfera celeste. Técnicamente, en astrología judiciaria cada una de estas casas representa un área de la vida ${ }^{7}$. El transcurso del movimiento diurno hace circular diariamente la esfera celeste y su contenido por las doce casas de modo que, dependiendo de la hora, la influencia de un elemento determinando se ejercerá principalmente sobre las circunstancias de la vida representadas por una u otra casa.

5 Probablemente, el término fue acuñado por Muhyī l-Dīn al-Magribī (m. 1283), cf. Dorce, C., El Tã̂y al-Azyā̂y de Muhyì l-Din al-Magribìi, Barcelona, 2002-2003, 63, 67-72; Kennedy, "The Astrological Houses", 555-557. Véase también Hogendijk, "The Mathematical Structure", 176-178 y nota 18.

${ }^{6}$ Cf. por ejemplo, Bouché-Leclercq, L'astrologie grecque, 256-286; Cátedra, P.M. (ed.) y Samsó, J. (intro.), El Tratado de Astrología atribuido a Enrique de Villena, Barcelona, 1983, 43-47.

7 Sobre los significados atribuidos tradicionalmente a cada una de las casas, pueden consultarse, por ejemplo, Bouché-Leclercq, L'astrologie grecque, 280-285; al-Bīrūnī, Kitāb al-tafhìm fì awà'il sinā'at al-tanŷ̀im, trad. inglesa de R. Ramsay Wright en al-Bīrūnī, The Book of Instruction of the Art of Astrology. Written in Ghaznah, 1029 A.D. Reproduced from Brit. Mus. MS Or. 8349, Londres, 1934, cf. 275-295. 
Puesto que los elementos considerados en la astrología medieval tienen órbitas muy cercanas a la eclíptica, el problema del trazado de las casas se reduce al de determinar la longitud $\lambda$ de los puntos de la eclíptica, llamados principios de las casas, que cruzan cada una de estas divisiones. Tradicionalmente, las casas se numeran en sentido inverso al del movimiento diurno, a partir de la división que produce la intersección de la mitad oriental del plano del horizonte con la eclíptica en $\lambda_{1}$ (véase la figura 4). A este punto, principio de la casa I, que se encuentra ascendiendo por el horizonte, se le denomina ascendente (al-țāli ). En el punto diametralmente opuesto de la eclíptica se encuentra el principio de la casa VII, $\lambda_{7}$ o grado descendente (al-gārib). En general, aunque no siempre, los métodos para la división de casas integran también en el conjunto de las casas las dos culminaciones, superior e inferior, de la eclíptica, es decir, sus intersecciones con el meridiano local. La superior $\left(\lambda_{10}\right)$ se denomina medio cielo (wasat

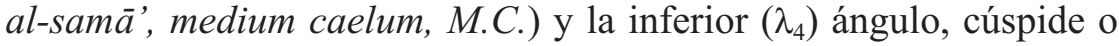
pivote de la tierra (watad al-ard, imum medium caelum, I.M.C., o antivertex). Quedando así definidos los principios de las cuatro casas conocidas como las cuatro cúspides (al-awtād al-arba'a).

Figura 4

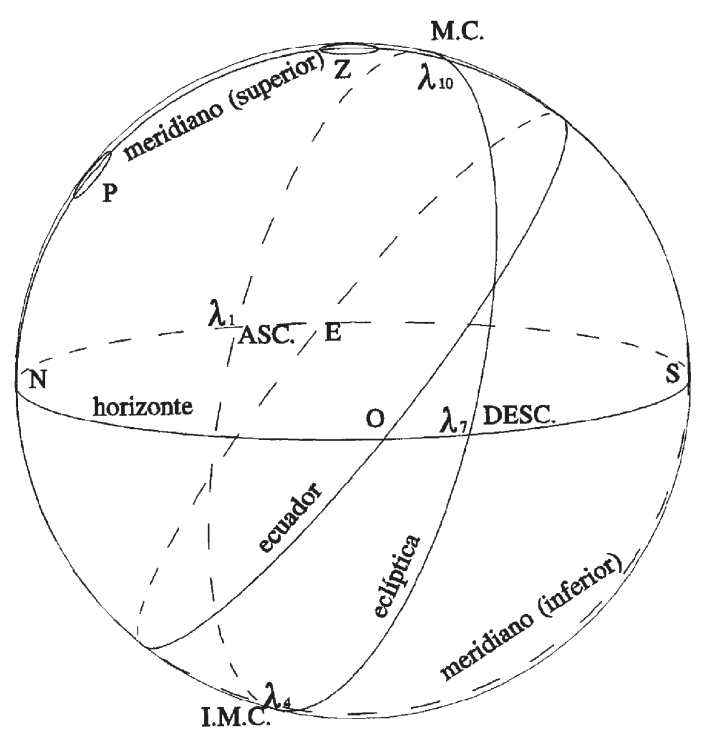

Al-Qanțara (AQ) XXX 1, enero-junio 2009, pp. 41-67 ISSN 0211-3589 
Sin embargo, el problema de la delimitación del resto de casas ha ido originando desde la antigüedad hasta nuestros días una diversidad de métodos que, ofreciendo resultados distintos, implican también un grado variable de habilidad matemática. En 1986, J.D. North ${ }^{8}$ elaboró, para los siete métodos que halló en fuentes antiguas o medievales, una clasificación que permite disponer de una denominación y definición establecida para cada uno de ellos. Posteriormente, E.S. Kennedy ${ }^{9}$ descubrió dos nuevos métodos y analizó la presencia de los distintos procedimientos en el islam medieval a partir de 28 fuentes árabes o persas. La siguiente lista contiene, en extracto, siguiendo la numeración establecida por North y la exposición elaborada por Kennedy, la descripción de los métodos de esta clasificación. En todos estos sistemas las casas se oponen respectivamente en cuadrantes opuestos, de modo que se cumple la condición

$$
\lambda_{\mathrm{i}}=\lambda_{\mathrm{i}+6 \text { (módulo 12) }}+180^{\circ} \text { (módulo } 360^{\circ} \text { ), }
$$

por lo que en las figuras se han representado únicamente las seis casas que se hallan por encima del horizonte.

\subsection{Método de las líneas horarias}

En este método, de límites fijos respecto al horizonte, los principios de las casas quedan determinados por las intersecciones de la eclíptica con las curvas de las líneas para las horas temporales pares ${ }^{10}$.

El modo de resolución más común para este método consiste en utilizar las líneas horarias trazadas en un astrolabio, con lo que la determinación de los límites de las casas es inmediata. En cambio, una solución exacta usando funciones trigonométricas es ciertamente compleja y requiere la solución de una ecuación de tercer grado ${ }^{11}$.

Kennedy no halla el procedimiento en ninguna de las fuentes que utiliza en el mencionado trabajo ${ }^{12}$. Sin embargo, una lámina de astro-

${ }^{8}$ North, Horoscopes and History, 46-47.

9 Kennedy, "The Astrological Houses"; North, "A Reply"; Hogendijk, "Progressions", § 5.3.1.

${ }^{10}$ Cf. North, Horoscopes and History, 20-27.

11 Ibidem, 23; Hogendijk, "Progressions", § 5.3.1: nota 52.

12 Kennedy, "The Astrological Houses", 538.

Al-Qanțara (AQ) XXX 1, enero-junio 2009, pp. 41-67 ISSN 0211-3589 
labio de 1304-1305, obra del granadino Ahmad b. Husayn b. Bāṣo ${ }^{13}$, considerada como destinada al método estándar (véase $\S 4.1$ ) puede relacionarse mejor con este método ${ }^{14}$. Además, el procedimiento está documentado en varios tratados andalusíes de astrolabio y, en opinión de Hogendijk ${ }^{15}$, el mismo planteamiento geométrico puede corresponder al sistema de casas usado por Māšā'allāh ( $c a$. 850), donde el cálculo se resolvería por procedimientos aritméticos en los que intervienen proporciones de la diferencia ascensional $(\Delta \alpha)$ correspondiente a la longitud eclíptica del principio de cada casa. Por otra parte, Ibn $M u$ 'ād de Jaén, a pesar de no referirse de un modo claro a la existencia de este procedimiento cuando dedica varios pasajes de su Risāla fi mațrạ al-šs ' $\bar{a}$ ' $\bar{t} t$ a realizar una revisión crítica de los métodos para dividir las casas, alude a la posibilidad de efectuar divisiones en la eclíptica dependiendo «del momento del paso del astro por cada parte - o aproximadamente - $(\mathrm{cf}$. $§ 6.1$ ), con lo que parece tener en cuenta la existencia de algún sistema que relaciona las divisiones sobre la eclíptica con fracciones de tiempo o aproximaciones a estas fraccio-

13 Sobre los problemas que plantea la identificación de los personajes de esta família, véase el estudio de E. Calvo en Ibn Bāṣo, Abū 'Alī al-Husayn, Risālat al-șafịha al-ŷami 'a li-ŷamì' al-'urụ̈̂ (Tratado sobre la lámina general para todas las latitudes), Madrid, 1993, 23-25.

${ }^{14}$ Cf. Kennedy, "The Astrological Houses", 574. La lámina, que contiene la inscripción "método de Ptolomeo", fue publicada junto a otra similar (sin la mención de Ptolomeo) perteneciente al mismo astrolabio por North (Horoscopes and History, 62-63). Ambas láminas contienen en su grabado una numeración correspondiente a las casas astrológicas distribuidas sobre las líneas de las horas temporales pares y en sentido antihorario, por lo que parece sensato considerar que, en el caso que nos ocupa, nos hallamos simplemente ante un ejemplo más de la atribución del método de las líneas horarias a Ptolomeo ( $c a$. 150). A pesar de que en la obra conservada de este autor no se describe ningún método para dividir las casas, no es extraño en al-Andalus hallarlo relacionado con el método de las líneas horarias: la misma atribución aparece también en los tratados de astrolabio de Ibn al-Samh (m. 1035) y en el tratado alfonsí del astrolabio esférico, donde se relaciona el procedimiento con Ptolomeo y Veles (Vettius Valens, astrólogo griego que, dependiendo de los autores, puede fecharse entre los siglos II y VIII, véase Sezgin, F., Geschichte des Arabischen Schriftums bis ca. 430 H., Leiden, 1979, VII, 38-41). Cf. Hogendijk, "Progressions", § 5.3, donde se citan Seeman, H., "Das kugelförmige Astrolab nach den Mitteilungen von Alfons X von Kastilien und den vorhandenen arabischen Quellen", en Abhandlungen zur Geschichte der Naturwissenschaften und der Medizin, Erlangen, 1925, VIII, 28 (reimp. en F. Sezgin (ed.), Arabische Instrumente in orientalistischen Studien, Frankfurt, 1991, IV, 390) y Viladrich, M., El "Kitāb al-'amal bi-l-asturlāb" (Llibre de l'ús de l'astrolabi) d'Ibn al-Samh, Barcelona, 1986, 67: nota 140 (comentario) y 124 (traducción); véase también Rico, M., Libros del Saber de Astronomía del rey D. Alfonso X de Castilla, Madrid, 1863, II, 193.

15 Cf. Hogendijk, "Progressions", § 5.3.

Al-Qanțara (AQ) XXX 1, enero-junio 2009, pp. 41-67 ISSN 0211-3589 
nes, lo que encajaría con el uso de procedimientos documentados para la aplicación de este método usando reglas aritméticas (véase $\S 4.8$ ). Los astrólogos modernos atribuyen el sistema a Placidus (Placido de Tito, m. 1668) y es el método con el que se han calculado las famosas tablas de Raphael (seudónimo de Robert Cross Smith, 1795-1832).

\subsection{Método estándar}

Una vez determinadas las cuatro cúspides mediante las intersecciones de la eclíptica con el horizonte y el meridiano local, en cada uno de los cuadrantes así obtenidos se divide el tramo de ecuador celeste comprendido entre las ascensiones rectas $\left(\alpha_{0}\right)$ de las dos cúspides que lo delimitan en tres secciones iguales. Los puntos en que los círculos meridianos que pasan por estas divisiones cruzan la eclíptica definen los límites de las restantes casas. En este caso la arista común utilizada es el eje del mundo. La figura 5 consiste en una proyección cenital de la esfera celeste (en la que el círculo NESO es el horizonte) y en ella se representan las seis casas que se hallan por encima del horizonte en un momento determinado.

Figura 5

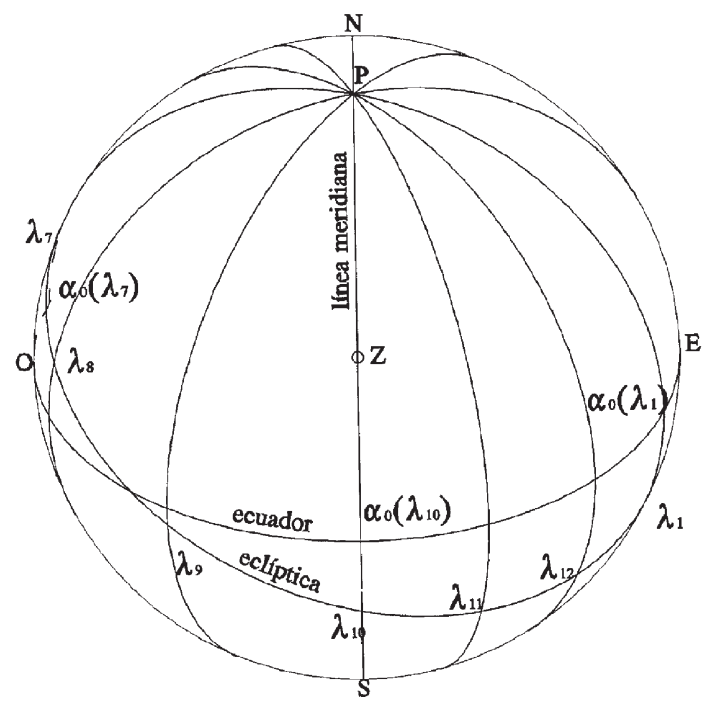

Al-Qantara (AQ) XXX 1, enero-junio 2009, pp. 41-67 ISSN 0211-3589 
El procedimiento no es de límites fijos. Con el transcurso del día, los límites de las ocho casas que no son cúspides cambian su posición con respecto al horizonte.

Todas las fuentes analizadas por Kennedy, excepto una, contienen referencias a este método y es el único sistema para el que encuentra en estas fuentes tablas completas. A pesar de su moderna atribución a Alcabitius (al-Qabīṣī, fl. Alepo, ca. 950) ${ }^{16}$, el origen del sistema es incierto. Se tiene constancia de él desde tiempos preislámicos ${ }^{17} \mathrm{y}$, en opinión de North ${ }^{18}$, puede ser anterior al resto de procedimientos.

En al-Andalus, el método se relaciona frecuentemente con Ptolomeo, aunque su descripción no se halla en las obras conservadas de este autor. Entre los textos sobre instrumental astronómico que describen el procedimiento, esta atribución se produce en el tratado de la azafea zarqāliyya de Azarquiel, el de la lámina universal de 'Alī b. Jalaf, el de la lámina general de Ibn Bāṣo y en el Libro Segundo de las Armellas alfonsí, donde el método se atribuye a Ptolomeo y a al-Battānī ${ }^{19}$. Ibn Mu'ād de Jaén rechaza explícitamente la utilidad de este método, tanto en el capítulo 52 de sus Tablas de Jaén, donde precisa que toma la descripción de al-Jwārizmīi ${ }^{20}$ (fl. ca. 830), como en su Risāla fì mațraḥ al-šu 'ā 'ät, don-

${ }^{16}$ En su Introducción a la astrología, al-Qabīṣī se limita a indicar que cada uno de los "cuatro cuadrantes" que definen en la eclíptica sus intersecciones con el horizonte y el meridiano se divide en tres partes desiguales "dependiendo de las ascensiones oblicuas del ascendente, de modo que el círculo queda dividido en doce partes llamadas casas [...]", y asegura que el modo de llevar a cabo esta operación "se explica en las tablas astronómicas", sin dar más detalles relativos al cálculo, cf. Burnett, Ch., Yamamoto, K. y Yano, M., al-Qabiși (Alcabitius): The Introduction to the Astrology. Editions of the Arabic and Latin texts and an English translation, Londres-Turín, 2004, 46-47. La referencia al uso de las ascensiones oblicuas del ascendente encaja mejor con el procedimiento aritmético usado por Māšāalalāh al que me he referido en el apartado anterior que con el método estándar. Sobre al-Qabīṣī, véase también Pingree, D., "al-Qabīṣi”, en Ch. C. Gillispie (ed.), Dictionary of Scientific Biography, Nueva York, 1975, XI, 226; Sezgin, F., GAS, VII, 170-171.

${ }_{17}$ Cf. Kennedy, “The Astrological Houses", 539-540; Hogendijk, "Progressions", § 5.4; North, Horoscopes and History, 6, 72; Neugebauer, O. y van Hoesen, H.B., Greek Horoscopes, Memoirs of the American Philosophical Society, Philadelphia, 1959, 48, 138-140.

18 North, "A Reply", 580.

${ }^{19}$ Cf. Calvo, "La résolution graphique", 35-36.

20 Cf. Ibn Mu'ād, Scriptum antiquum saraceni cuiusdam, de diversarum gentium Eris, annis ac mensibus, et de reliquis Astronomiae principiis, Nüremberg, 1549, cap. 25 (ed. de la trad. latina de Gerardo de Cremona de las Tablas de Ibn Mu'ā al-Ŷayyānī); Samsó, J., Las Ciencias de los Antiguos en al-Andalus, Madrid, 1992, 157-158; Castells, M. y Samsó, J., "Seven Chapters of Ibn al-Saffār's lost Zïj", Archives Internationales d'Histoire des Sciences, 45 (1995), 243-244; Neugebauer, O., The Astronomical Tables of al-Khwärizmī. Translation with Commentaries of the Latin Version edited by H. Suter

Al-Qanțara (AQ) XXX 1, enero-junio 2009, pp. 41-67 ISSN 0211-3589 
de dedica al procedimiento el pasaje que traduzco a continuación (véase la edición del texto árabe $\S 6.2$ ) y que incluye una singular referencia al método ecuatorial de límites móviles (cf. § 4.6):

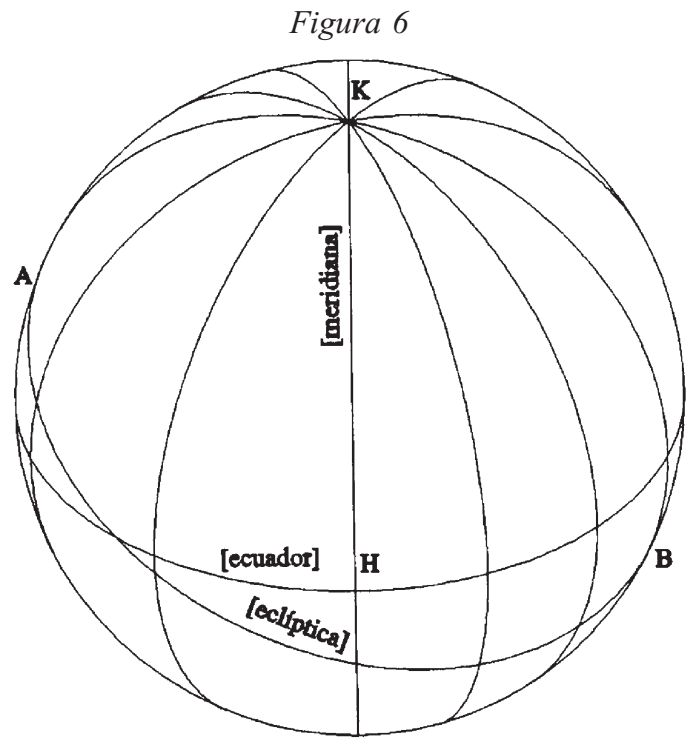

«Por lo que respecta a la división que se atribuye a Ptolomeo, funciona de otro modo y consiste - dicen- en que debemos conocer qué punto del ecuador está en el mismo meridiano que el punto $B^{21}$ trazando, desde el polo $K$, un arco hacia el punto $B$. Dividen el arco comprendido entre la intersección [de este meridiano] con el ecuador y el punto

supplemented by Corpus Christi College MS 283, Copenhagen, 1962, 76-78, 128-129; Suter, H., Die astronomischen Tafeln des Muhammed ibn Mūsā al-Khwārizmī in der Bearbeitung des Maslama ibn Ahmed al-Madjrịți und der lateinischen Übersetzung des Adelard von Bath auf Grund der Vorarbeiten von A. Bjørnbo und R. Besthorn herausgegeben und Kommentiert von Kongelige Danske Videnskabernes Selskab, Copenhague, 1914 (reimp. en H. Suter, Beiträge zur Geschichte der Mathematik und Astronomie im Islam, F. Sezgin (ed.), Frankfurt, 1986, I, 25, 29-30, 96-98, 194-205).

21 Véase la figura 6, inexistente en el manuscrito. Para la transcripción de letras que simbolizan puntos en esta figura, utilizo las equivalencias $A=1, B=\varphi, H={ }_{\tau}, K=$ s, de acuerdo con el sistema propuesto por Kennedy: Kennedy, E.S. y Hermelink, H., "Transcription of Arabic Letters in Geometrical Figures", JAOS, 82, 2 (1962), 204; Kennedy, "Colleagues and former students", 745. 
$H$ en tres partes y trazan un arco desde $K$ hasta cada una de estas divisiones. Las partes que separan esos arcos sobre la eclíptica son las tres casas orientales [que se hallan por encima del horizonte]: la de los enemigos [XII], la de la felicidad [XI] y la del poder [X]. Del mismo modo operan en la región occidental, a partir del punto $A$. Pero no sé qué les llevó por este camino ni qué lógica les empujó hacia este método, si no es algo insostenible, en mi opinión. Ello se debe a que, si trasladas este procedimiento de división a los lugares ecuatoriales, donde el círculo del ecuador es vertical, y no inclinado, los arcos divisorios se trazarán desde el punto en que se encuentran el horizonte y el meridiano, pues ocurre allí que este punto es el polo norte o el sur. Pero cuando se cambia el lugar en cuestión por sitios que tienen latitud no cambian el punto de donde parten los arcos y se ponen a trazarlos siempre únicamente desde el polo, esté donde esté. No veo que este modo de dividir tenga ninguna propiedad que sea útil excepto que el grado del ascendente, en su avance siguiente en el tiempo, pasa por estos arcos que se han trazado de modo que llega a ellos en períodos de tiempo iguales, y que el grado del descendente haya pasado antes por los arcos occidentales en tiempos [también] iguales. ¿Qué utilidad hay en esto?, es más, ¿qué tiene que ver este objetivo con el que se busca? Si fuera esto [74v] lo que conviene tener en cuenta al dividir, debería desprenderse obligatoriamente de su discurso que el arco que se traza desde el punto $K$ hacia $B$ y el otro arco, que se traza desde el polo $K$ hacia el punto $A$, produzcan una división del ecuador en partes iguales, con porciones del ecuador obtenidas por medio de arcos trazados desde el polo $K$. Si esto fuera así no entraría en contradicción con lo que dicen, pero la división de la casa del poder $[\mathrm{X}]$ no coincidiría con el punto $H$, y esto no lo dicen para nada. [En cambio,] conviene que la división en su totalidad - es decir, las doce casas - tenga una base única y consecuente, ya que sobre ella se sustentan todas las casas y se dividen por ese sistema las cuatro casas correspondientes a la vida [I], a los descendientes [IV], al matrimonio [VII] y al poder [X], que son conocidas como las cúspides. Por lo que respecta a determinar algunas de ellas de un modo cualquiera y luego, si queremos dividir las restantes utilizar otro método, no hay lógica en ello a menos que surja algo que sea consecuencia de esa división que representan las cuatro cúspides. No creo que este método sea satisfactorio y no considero bueno que lo siga aquel que prefiere la verdad y da vida a la ciencia». 


\subsection{Método de las dos longitudes}

En este método, los arcos de la eclíptica que se hallan entre dos cúspides se dividen en tres partes iguales que corresponden a las casas.

Atribuido a veces a Porfirio de Tiro (ca. 232-304), está documentado en fuentes griegas ${ }^{22}$ y al-Bīrūnī (m. 1048) lo denomina método de los antiguos $(a l-a w \bar{a} ' i l)$, mientras que los autores orientales posteriores lo atribuyen al Occidente islámico. Kennedy halla este método en siete de las fuentes que utiliza, siendo tres de ellas occidentales: el $Z \bar{y} \hat{y}$ de Ibn Ishạa al-Tūnisī (donde el método parece apropiado para asuntos ordinarios, dado que, para cuestiones importantes prescribe usar el método estándar) ${ }^{23}$, el al-Zîy al-Qawìm de Ibn al-Raqqām ${ }^{24} \mathrm{y}$ el segundo Zî̀ de Muhyī l-Dīn al-Magribī, quien lo atribuye a un autor del Magrib que no nombra ${ }^{25}$.

Recientemente, J. Samsó ha hallado el método usado sistemáticamente por Ibn Qunfud al-Qusantịnī (1339-1407) ${ }^{26}$ y ha recogido también la información contenida en una Risāla de 'Abd Allāh Aṣnāk al-Marrākušĩ (fl. ca. 1655) sobre el uso de las tablas del Almanaque Perpetuo de Abraham Zacuto (1452-1515), donde se describe el correspondiente modo operativo con un ejemplo numérico ${ }^{27}$. Finalmente, cabe considerar que, a continuación del mismo pasaje de la Risāla fì mațrah al-šu 'à 'àt de Ibn Mu'ād mencionado en relación al método de las líneas horarias (cf. § 4.0), aparece una referencia imprecisa a dividir la eclíptica «en partes iguales» (cf. § 6.1) que puede aludir a la utilización de este método o bien al método de la longitud única (véase $\S 4.6)$.

${ }^{22}$ Kennedy, "The Astrological Houses", 540; North, Horoscopes and History, 6, 72; Neugebauer y van Hoesen, Greek Horoscopes, 152-157.

${ }^{23}$ Cf. Kennedy, "The Astrological Houses", 554-555; Mestres, A., "Maghribī Astronomy in the 13th century: a Description of Manuscript Hyderabad Andra Pradesh State Library 298", en Casulleras y Samsó (eds.), From Baghdad to Barcelona, I, 400-401.

${ }^{24}$ Kennedy, "The Astrological Houses", 557-558.

25 Ibidem, 557.

${ }^{26}$ Cf. Samsó, J., "Cuatro horóscopos sobre muertes violentas en al-Andalus y el Magrib", en M. Fierro (ed.), De muerte violenta. Política, religión y violencia en al-Andalus, EOBA, XIV, Madrid, 2004, 500-502.

${ }^{27}$ Cf. Samsó, J., “Abraham Zacut and José Vizinho's Almanach Perpetuum in Arabic (16th-19th C.)", Centaurus, 46 (2004), 87-88. 


\subsection{Método del primer vertical de límites fijos}

El círculo máximo que pasa por el cenit del lugar y por los puntos Este y Oeste del horizonte (véase la figura 7, el círculo NESO representa el horizonte), denominado primer vertical, queda dividido en cuatro cuadrantes por el meridiano y el horizonte. Trazando círculos de posición que pasen por los puntos Norte y Sur del horizonte y por divisiones uniformes del primer vertical se determinan, en sus intersecciones con la eclíptica, los límites de las doce casas. En este caso, la arista común es la línea meridiana (línea Norte Sur, sobre el horizonte local) y el procedimiento es de límites fijos, es decir, los límites de las doce casas no cambian su posición con respecto al horizonte.

\section{Figura 7}

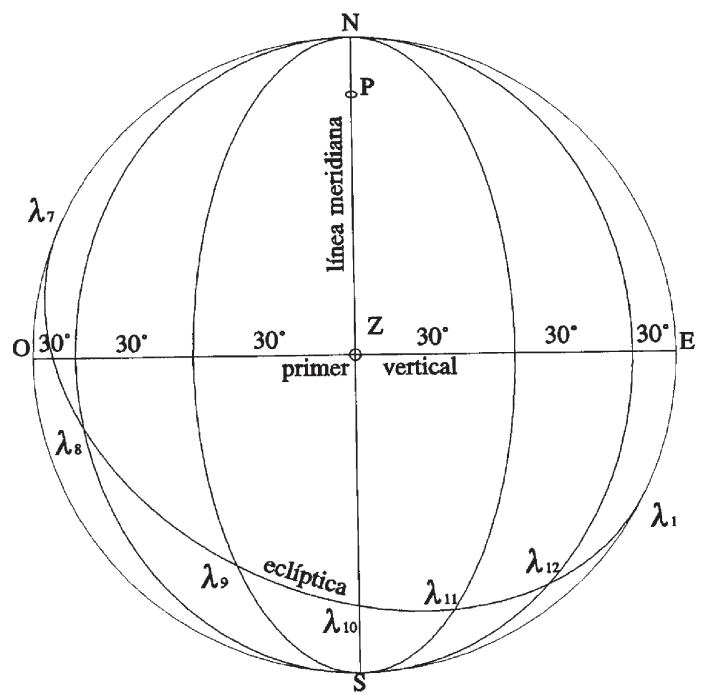

Doce de las fuentes que menciona Kennedy 28 incluyen este método. En Oriente se atribuye a al-Bīrūnī, quien ofrece tres soluciones distintas para resolverlo y asegura haberlo inventado ${ }^{29}$. En al-Anda-

${ }^{28}$ Cf. Kennedy, "The Astrological Houses", 541-543.

${ }^{29}$ Lo califica de "el método que prefiero" (al-tarīq allad̄i ātartu-hu) en su Qānūn (cf. al-Bīrūnī, al-Qānūn al-Mas 'üdì, Hyderabad, 1956, III, 1359-1369; North, Horosco- 
lus, sin embargo, los algoritmos aplicados a su resolución son distintos de los orientales y se atribuye en numerosas fuentes al mítico Hermes, por lo que, sin descartar la posibilidad de una transmisión de este método desde Oriente, Kennedy supone que el sistema puede ser de origen preislámico y que las distintas soluciones pudieron haberse desarrollado de modo independiente. La primera referencia al método en el contexto andalusí corresponde a un Zîy perdido de Ibn al-Samh, donde ya aparece vinculado a Hermes. Ibn Mu'ād de Jaén, que rechaza de forma expresa la utilidad de este método y manifiesta su incredulidad acerca de su supuesta atribución a Hermes, nos transmite en su Risāla fì mațraḥ al-šsu 'áa 'ât el pasaje del Zîy de Ibn al-Samḥ donde se describe un procedimiento erróneo para su cálculo y aporta además una solución correcta ${ }^{30}$. Posteriormente, el método se halla atribuido a Campanus (ca. 1210-1296) en el Oeste latino.

\subsection{Método ecuatorial de límites fijos}

El procedimiento, también de límites fijos, es análogo al del método anterior (véase la figura 8, el círculo NESO representa el horizonte). En este caso, la arista común de las casas es también la línea meridiana pero el círculo sobre el que se practica la división en secciones uniformes de $30^{\circ}$, mediante círculos de posición, es el ecuador celeste.

En el ámbito islámico, Kennedy solamente ha hallado el método en Occidente y la primera manifestación de este procedimiento la representa Ibn Mu'ād, quien ofrece, en dos de sus obras, Tablas de Jaén y Risāla fì Matrah al-šs 'a 'āt, un algoritmo exacto para resolverlo ${ }^{31}$. El sistema aparece también en tres tratados alfonsíes sobre instrumental

pes and History, 32-33; Samsó, J., ““‘al-Bīrūn̄” in al-Andalus”, en Casulleras y Samsó (eds.), From Baghdad to Barcelona, II, 588) y especifica que el método es de su propia creación y lo denomina "mi procedimiento" (madhabi) en sus Maqālìd y en su Isti 'äb (cf. Kitāb maqālìd 'ilm al-hay'a. La Trigonométrie sphérique chez les Arabes de l'Est à la fin $d u X^{e}$ siècle, M.-Th. Debarnot (ed. y trad.), Damasco, 1985, 284-285; Kitāb fì isti 'āb al-wuŷūh al-mumkina li-șan 'at al-asțurlāb, MSS Istambul Carullah, 1451, fol. 24r, Biblioteca Nacional de Túnez, 5540, fol. 35r; Samsó, "al-Bīrūnī”, 589).

${ }_{30}$ Ms. Florencia, Medicea Laurenziana, Or 152, fols. $75 r-76 v$. Cf. Kennedy, "Ibn Mu'ādh", 157-160 e idem, "The Astrological Houses", 541, 543, 552-554.

${ }^{31}$ Cf. Hogendijk, "Applied Mathematics", 88-89, 92-99, 107-109 (texto latino), 110-112 (traducción).

Al-Qantara (AQ) XXX 1, enero-junio 2009, pp. 41-67 ISSN 0211-3589 
Figura 8

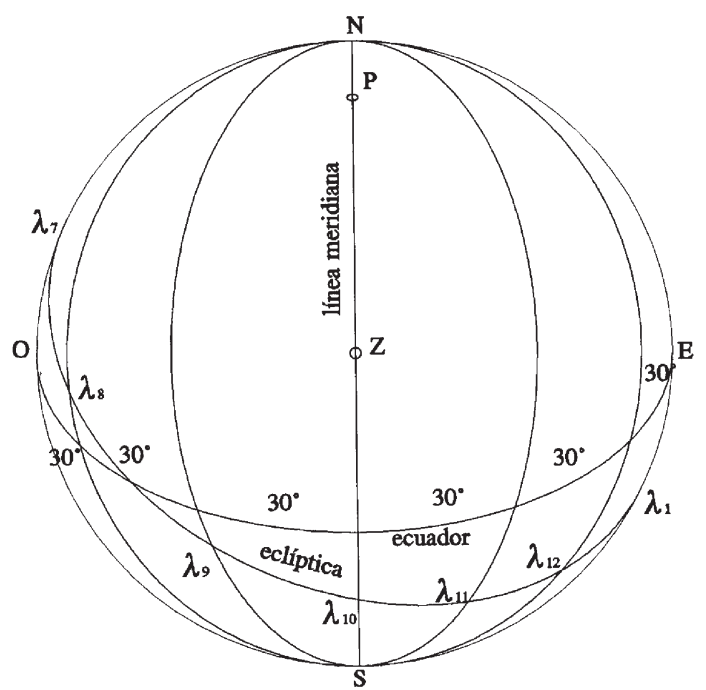

astronómico, donde se relaciona con Ibn $\mathrm{Mu}^{\prime} a \bar{a} \underline{\mathrm{d}}$, Hermes y Azarquiel ${ }^{32}$. Sin embargo, posteriormente, en el Oeste latino se atribuyó el método a Regiomontano, quien poseyó un manuscrito de las Tablas de Jaén ${ }^{33}$.

Con anterioridad a Ibn $\mathrm{Mu}^{6} \mathrm{a} \underline{\mathrm{d}}$ se hallan procedimientos análogos al método ecuatorial para dividir las casas aplicados a las doctrinas astrológicas de la proyección de rayos y del tasyīr. En opinión de Samsó ${ }^{34}$, esto permite suponer que, aunque pudo haber elaborado su propio algoritmo de cálculo para resolverlo, Ibn Mu'ād no inventó el método ecuatorial sino que aplicó a la división de casas métodos ya existentes para otras prácticas astrológicas. Tras la lectura de la Risāla

32 Libro Segundo de las Armellas, Libro dell Ataçir (en ambos con atribución a Ibn Mu'ād) y Libro dell Astrolabio Redondo (con atribución a Hermes y Azarquiel). Cf. Calvo, "La résolution graphique", 36; Hogendijk, "Progressions", § 5.1. Ambos están de acuerdo en corregir la identificación que propone North (Horoscopes and History, 34) de la descripción contenida en el tratado alfonsí del astrolabio esférico con el método del primer vertical. Véase también Samsó, "al-Bīrūnī”, 594.

33 North, J.D., "The Alfonsine Books and some Astrological Techniques", en M. Comes, R. Puig y J. Samsó (eds.), De Astronomia Alphonsi Regis. Actas del Simposio sobre Astronomía Alfonsí celebrado en Berkeley (agosto 1985) y otros trabajos sobre el mismo tema, Barcelona, 1987, 49.

34 Cf. Samsó, "al-Bīrūnī”, 594.

Al-Qanțara (AQ) XXX 1, enero-junio 2009, pp. 41-67 ISSN 0211-3589 
fì mațrạ̣ al-šs 'a'a $\bar{a} t$, puede reforzarse esta opinión con la del propio Ibn $\mathrm{Mu}^{\prime} a \bar{d}$, quien, sin atribuirse en ningún momento la invención del método, se declara partidario de excluir cualquier otro procedimiento e insiste en que las doctrinas de la proyección de rayos y la división de casas son inseparables, se deben abordar desde una misma base teórica y debe aplicárseles un mismo método ${ }^{35}$.

\subsection{Método ecuatorial de límites móviles}

Partiendo de $\alpha_{0}\left(\lambda_{1}\right)$, se divide el ecuador celeste en doce partes iguales de $30^{\circ}$ cada una (vease la figura 9, el círculo $N E S O$ representa el horizonte), los meridianos que pasan por estas divisiones determinan, al cruzarse con la eclíptica, los límites de las doce casas. Al igual que en el método estándar, la arista común es el polo del mundo. Los límites de las casas no son fijos con respecto al horizonte y, además,

${ }^{35}$ La idea aparece de modo recurrente en varios pasajes del tratado. Así, por ejemplo, en el fol. $72 v$ (ms. Florencia, Medicea Laurenziana Or 152) se afirma que "[...] nosotros, a partir de la división de la esfera celeste en sus casas, queremos entrar en el asunto de la proyección de los rayos, ya que los dos temas van por un camino único y no diverso [...]", en el $77 r$, con la ayuda de referencias a una figura (inexistente en el manuscrito, que no contiene ilustraciones) se expresa lo que equivale a afirmar que un astro situado en el principio de una de las casas tendrá sus trígonos, cuadraturas y sextiles, respectivamente, a una distancia de cuatro, tres y dos casas, mientras que en el $77 v$, refiriéndose claramente al método ecuatorial de límites fijos, Ibn $\mathrm{Mu}$ 'ād declara que "[...] lo que consideramos correcto - y prescindimos de cualquier otro procedimiento - consiste en dividir el círculo del ecuador en partes iguales - puesto que es el origen y la dirección del movimiento - y en que los círculos divisorios, para cualquier clima, se tracen desde los dos puntos comunes a su horizonte y a su meridiano local. Esto es lo que expresa nuestra opinión y nuestro empeño y lo mismo pretendió Ibn al-Samh en lo que atribuyó a Hermes acerca de la proyección de rayos concretamente [...]" y, poco más adelante, asegura que "[...] refuerza nuestra opinión y nuestra creencia acerca de agrupar [en una misma teoría] la división de las casas y la proyección de los rayos el hecho de que al-Kindī, que fue de tal modo excelso en esta clase de ciencias que es de todos conocido, dice en una de sus obras sobre los rayos que, si el astro está en el círculo del horizonte, en el grado del ascendente, el grado de la casa XI y el grado de la casa III son sus dos sextiles, el grado del medio cielo y la culminación inferior son sus dos cuadraturas y el grado de la casa IX y el grado de la casa $\mathrm{V}$ son sus dos trígonos, y esto no es posible de ningún modo en el método que se atribuye a Ptolomeo. Ya hemos demostrado la inutilidad de ese método. En cambio, sí es posible de acuerdo con lo que hemos referido acerca de dividir el ecuador en partes iguales y, luego, trazar por esas divisiones círculos desde los dos puntos comunes al horizonte y al meridiano [local]. Esos círculos dividen la eclíptica en sus doce casas y cualquier astro situado sobre uno de esos círculos tiene [respectivamente] su trígono, su cuadratura y su sextil a una distancia de cuatro, tres y dos casas [...]" (cf. traducción inglesa de este último pasaje en Hogendijk, "Applied Mathematics", 102).

Al-Qantara (AQ) XXX 1, enero-junio 2009, pp. 41-67 ISSN 0211-3589 
Figura 9

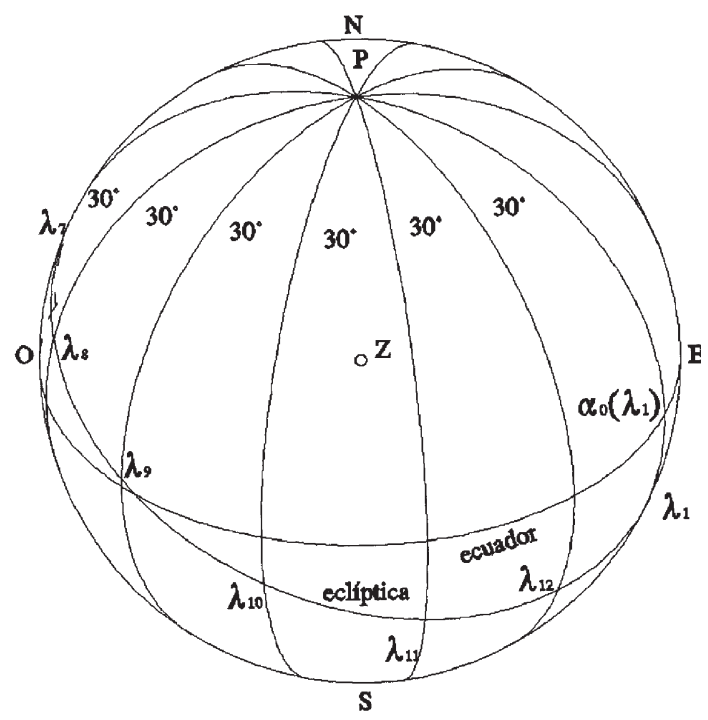

las casas X y IV sólo coinciden con el meridiano cuatro veces al día, cuando el ascendente es $0^{\circ}, 90^{\circ}, 180^{\circ}$ o $270^{\circ}$.

North ${ }^{36}$ llama la atención sobre la existencia de esta técnica, adscrita a Ptolomeo por Valentín Nabod (s. XVI) entre otros y considera que tal vez pudo originarse a causa de una mala interpretación del algoritmo que describe Ibn Mu'ād para el método 4 (ecuatorial de límites fijos), tal como se halla en las Tablas de Jaén.

Ninguna de las fuentes estudiadas por Kennedy contiene este método. En el tratado sobre proyección de rayos de Ibn Mu'ād, aparece únicamente como remate de la refutación que realiza del método estándar (cf. traducción del pasaje en § 4.1, supra), sin referencia alguna a que se aplique en la práctica, por lo que puede tratarse tanto de una simple especulación por parte del autor como de una alusión a un método realmente utilizado, con lo que nos encontraríamos ante la única mención conocida de este método en el ámbito islámico.

36 North, Horoscopes and History, 42.

Al-Qanțara (AQ) XXX 1, enero-junio 2009, pp. 41-67 ISSN 0211-3589 


\subsection{Método de la longitud única}

Denominado así debido a que todas las casas tienen el mismo grado de longitud dentro del signo al que pertenecen. Consiste en tomar como casas doce divisiones de $30^{\circ}$ directamente sobre el círculo de la eclíptica a partir del grado ascendente. En este caso, la arista común es el eje de la eclíptica. Al igual que ocurre con el método anterior, se trata de un método de límites móviles con respecto al horizonte y que no respeta la condición de que las casas X y IV coincidan permanentemente con el meridiano. El método está documentado en horóscopos griegos ${ }^{37} \mathrm{del}$ siglo V y no aparece en ninguna de las fuentes que utiliza Kennedy 38 .

Como se ha dicho (cf. § 4.2), Ibn Mu'ād puede aludir a este sistema de división, o tal vez a su variante de las dos longitudes, cuando se refiere simplemente a dividir la eclíptica «en partes iguales» (cf. $\S 6.1)$.

\subsection{Método de Habaš}

Añadido a la lista por Kennedy ${ }^{39}$, únicamente está documentado en una obra de al-Bīrūnī ${ }^{40}$, quien lo atribuye a Habaš (al-Hāàib, $f l$. 850). La particularidad del método consiste en que divide la eclíptica a partir de divisiones realizadas sobre el horizonte. Una vez halladas las cuatro cúspides del modo habitual, se dividen en tres partes iguales cada uno de los arcos de acimut (arcos del horizonte) que se hallan entre el ascendente o el descendente y los puntos norte y sur del horizonte. La proyección de estas divisiones sobre la eclíptica mediante arcos de círculos de altura (círculos máximos que pasan por el cenit del lugar) determina los principios de las casas.

${ }^{37}$ Cf. North, Horoscopes and History, 6 y 72; Neugebauer y van Hoesen, Greek Horoscopes, 149-150.

${ }^{38}$ Kennedy, "The Astrological Houses", 544.

39 Ibidem, 544, 549-550.

40 Al-Bīrūnī, Kitāb maqālìd, 280-281.

Al-Qanțara (AQ) XXX 1, enero-junio 2009, pp. 41-67 ISSN 0211-3589 


\subsection{Método de las diferencias divididas (Split Differences)}

Añadido también a la lista por Kennedy y solamente hallado en el al-Zīy al-Šămil de Ibn al-Raqqām (m. 1315) ${ }^{41}$. El texto analizado por Kennedy prescribe dividir el cuadrante del ecuador celeste comprendido entre el punto Este del horizonte y el meridiano en tres partes y hallar la diferencia de longitudes, usando $\alpha_{0}^{-1}$ y $\alpha_{\varphi}^{-1}$, que corresponde a cada uno de los dos puntos de división, de modo que se obtendrán dos segmentos en la eclíptica. El principio de la casa XI estará situado en el segmento superior, a un tercio de distancia desde la longitud hallada usando $\alpha_{0}^{-1}$ hacia la longitud hallada usando $\alpha_{\varphi}^{-1}$. El principio de la casa XII estará situado en el segmento inferior, a un tercio de distancia desde la longitud hallada usando $\alpha_{\varphi}{ }^{-1}$ hacia la longitud hallada usando $\alpha_{0}{ }^{-1}$. El resto de casas se hallará de modo análogo.

En opinión de North ${ }^{42}$ el procedimiento parece ser un método aproximativo y Hogendijk ${ }^{43}$ concluye que consiste en un modo aproximado de calcular las casas de acuerdo con el método de las líneas horarias y, dado que en el tratado de uso del astrolabio de Ibn al-Samh se atribuye este método a Ptolomeo a través de Habaš al-Hāsib, propone que tal vez este último autor usara el mismo procedimiento de cálculo que Ibn al-Raqqām ${ }^{44}$.

Si tenemos en cuenta el citado pasaje de la Risāla fì matrah $a l-\check{s} u$ ' $\bar{a}$ ' $\bar{a} t$ de Ibn $\mathrm{Mu}$ 'ād en que se alude a la posibilidad de efectuar divisiones en la eclíptica dependiendo «del momento del paso del astro por cada parte —o aproximadamente— $\longrightarrow$ (cf. $\S \S 4.0$ y 6.1), bien puede entenderse que esta referencia al carácter aproximado del sistema corresponde al uso de un procedimiento para calcular las casas según el método 0 , de las líneas horarias, como el que aquí se ha definido. Por otra parte, está claro que el uso de proporciones establecidas sobre arcos de la eclíptica obtenidos a partir de la diferencia de longitudes hallada al proyectar puntos del ecuador usando $\alpha_{0}^{-1}$ y $\alpha_{\varphi}{ }^{-1}$ es una técnica conocida por Ibn $M u^{\prime} a \bar{d}$, dado que la utiliza en la misma

${ }^{41}$ Kennedy, "The Astrological Houses", 544-545, 568.

42 North, "A Reply", 580.

${ }^{43}$ Hogendijk, "Progressions", § 5.3.1.

${ }^{44}$ Cf. Hogendijk, "Progressions", §§ 5.3, 6.2.4; Viladrich, El "Kitāb al- 'amal”, 67 (comentario) y 124 (traducción).

Al-Qanțara (AQ) XXX 1, enero-junio 2009, pp. 41-67 ISSN 0211-3589 
Risāla al desarrollar el algoritmo aproximado para la proyección de rayos con que acaba el tratado ${ }^{45}$.

\section{Conclusiones}

El anterior repaso de los procedimientos de cálculo aplicados a la división de casas tiene dos consecuencias fácilmente verificables. Por una parte, al observar el conjunto de fuentes que hemos visto a la luz de la clasificación de estos métodos, se hacen patentes ciertas peculiaridades de una tradición astrológica occidental: tal es el caso de la aplicación a la división de casas del método ecuatorial de límites fijos, no documentado en Oriente, o el hecho de que en al-Andalus algunos procedimientos reciben atribuciones propias, como las que relacionan a Ptolomeo con los métodos estándar y de las líneas horarias, o al mítico Hermes con el método del primer vertical. Por otra parte, al trabajar con estos materiales, surge la notable certeza de que la propia clasificación de métodos se ha convertido en herramienta imprescindible para el estudio de fuentes relacionadas con la división de casas, dado que evita referencias imprecisas a su contenido por falta de denominaciones concretas para los procedimientos de cálculo que en ellas se muestran. La mera existencia de una clasificación de estas características puede considerarse no solamente un avance científico, sino a la vez un importante incentivo para la investigación de este tipo de materiales, en la medida en que ayuda enormemente a su comprensión y a situarlos en un conjunto bien referenciado. Éste es tal vez el motivo por el cual los estudios de nuevas fuentes con contenido astrológico, tradicionalmente ignoradas o consideradas con cierto recelo desde el ámbito académico, han proliferado considerablemente desde que se publicó, en 1986, la primera clasificación establecida por el profesor J.D. North, de quien lamentamos aquí su reciente fallecimiento.

45 Casulleras, "Ibn Mu‘ādh”, 392-402.

Al-Qantara (AQ) XXX 1, enero-junio 2009, pp. 41-67 ISSN 0211-3589 
6. Apéndice: dos fragmentos de la Risāla fì matrah al-šu ' $\bar{a}{ }^{`} \bar{a} t$ de Ibn Mu'ād

6.1. MS Florencia, Medicea Laurenziana, Or 152, fol. $73 r$

فليكن فلك البروج عليه اب وفلك الأفق المقاطع له فلك ابد و الظاهر من فلك البروج طائفة اهب و هو نصفه. فإذا أردنا أن نزداد من هذه القسمة طالبنا أنفسنا عن حقيقة ما تريد هذه القسمة وأيّ الاغر اض نقصد بها لتكون قسمتها مغرضة نحو ذلك الغرض و عملنا فيها تابعا لذلك المر اد فانت القسمات كثيرة وأغراضها شتى. فإناّ لو أردنا أن نقسم قوس اهب قسمة غرضنا مثلا أن نعلم الأطول من الأقصر : إمّا لنقف من ذلك على زمان مرور الكوكب بقسم قسم أو نحو ذلك إمّا تعدينا أن نجعل القسمة منصفة لقوس اب على السواء. وقد تقدّت هذه القسمة في قسمة جميع الفلك على يب [اثني] عشر جزءا أو سص درجة ولذلك رسمت درج السواء.

Figura 10

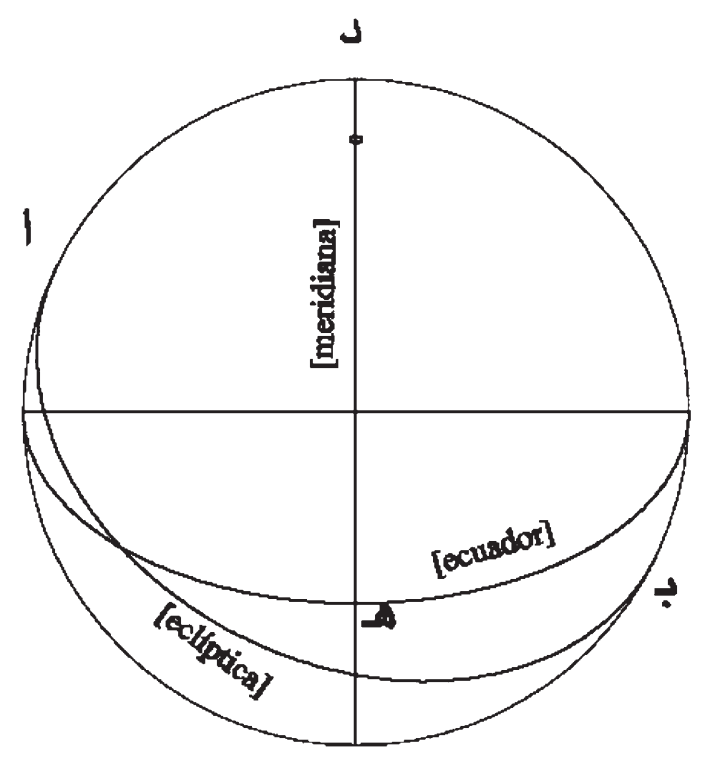

Al-Qanțara (AQ) XXX 1, enero-junio 2009, pp. 41-67 ISSN 0211-3589 


\section{Traducción}

Sea la eclíptica $A B^{46}$, el horizonte que la corta $A B D$ y la parte visible de la eclíptica $A E B$, que es su mitad. Si queremos proseguir con esta división, nos plantearemos qué es lo que en realidad se persigue con ella y cuál es el objetivo que queremos obtener, para que el modo de dividir utilizado esté orientado hacia ese objetivo y operaremos de este modo de acuerdo con dicho propósito, pues las maneras de dividir son muchas y sus finalidades son variadas. Si quisiéramos dividir el arco $A E B$ de modo que deseáramos, por ejemplo, distinguir lo más largo de lo más corto: o bien lo hacemos depender del momento del paso del astro por cada parte $-\mathrm{O}$ aproximadamente-, o bien procedemos a dividir el arco $A B$ en partes iguales. Hemos anticipado esta última técnica para dividir al referirnos a la división de toda la eclíptica en 12 partes, o bien en 360 grados, y para ello he trazado los grados eclípticos ${ }^{47}$.

\subsection{MS Florencia, Medicea Laurenziana, Or 152, fols. $74 r-74 v^{48}$}

$$
\begin{aligned}
& \text { وأمّا القسمة التي } 49 \text { ينسبونها إلى بطلميوس فإتها على غير هذا الوجه) وذلك أنهم يقولون ينبغي } \\
& \text { أن نعلم أيّ نقطة من معدّل النهار تكون مع نقطة باء50 على فلك واحد من أفلاك نصف النهار } \\
& \text { بأن نخرج من قطب كاف قوسا إلى نقطة باء .فما قطع من معدل النهار قسموا من ذلك الوضع } \\
& \text { إلى نقطة الحاء بثلاثة أقسام وأخرجوا إلى كلّ قسم قوسا من الكاف .فما قطعت تلك القسيّ من } \\
& \text { فلك البروج فهي البيوت الثلاثة المشرقيّة :بيت الأعداء وبيت السعادة وبيت الملك .وكذلك يفعلون } \\
& \text { بجهة المغرب بنقطة ألف. ولا أدري ما حملهم على هذا51 السبيل ولا أيّ قياس أداهم إلى هذا } \\
& \text { المسلك إلا وجه ضعيف أحسب. وذلك أنك إذا حوّلت هذه القسمة في بلاد الاستواء حيث فلك }
\end{aligned}
$$

46 Véase la figura 10, inexistente en el manuscrito. Para la transcripción de letras que simbolizan puntos en esta figura, utilizo las equivalencias $A=1, B=\rfloor, D=\lrcorner, E=\diamond$, siguiendo el sistema propuesto por Kennedy y Hermelink, "Transcription of Arabic Letters", 745.

${ }^{47}$ No hay en el texto una alusión previa a los grados de la eclíptica ni a su división en doce signos. Tal vez debe entenderse que cita una obra anterior, aunque probablemente se refiere a alguna figura que no está en el manuscrito.

48 Véase la traducción de este pasaje en $\S 4.1$.

الذي ms التي 49

50 V́éase la tigura 11, inexistente en el manuscrito.

هذه 51 هذ 
>معدّل النهار 52/ منتصب غير مائل كانت القسيّ القاسمة مخرجة من النقطة التي اشترك فيها فلك الأفق مع فلك نصف النهار فيتقق هناك أن تكون تلك النقطة القطب الشماليّ أو الجنوبيّ. فلمّا انتقل الموضع المفروض إلى البلاد ذوات العروض لم بينقوا هم موضع القسيّ المخرجة وجعلوا

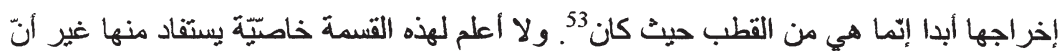
درجة الطالع إذا مرّت في المستقبل الآتي من الزمان بهذه القسيّ المخرجة انتهت اليها في أزمان متساوية وأنّ الدرجة الغاربة كانت قد مرّت قبل بالقسيّ الغربيّة في أزمان متساوية. وأيّ منفعة في هذا أو أيّ اشتر اك بين هذا الغرض وبين الغرض المطلوب. ولو كان هذا [74] هو الوجها الذي ينبغي أن نقسم به لوجب من قولهم ضرورة أن تكون القوس المخرجة من نقطة كاف إلى نقطة باء والقوس الاخرى المخرجة من قطب كاف إلى نقطة ألف تقسمان] ما بينه[م]ا من فلك معدل النهار أقساما متساوية بقسيّ مخرجة من قطب كاف]. و] لو كان هذا لا ينقض قولهم ووقع قسم بيث الملك على غير نقطة حاء وليس هذا من شيء من أقاويلهم. فإنما ينبغي بأن يكون لهذه القسمة كلها أعني البيوت الاثني عشر أصلا واحدا مطردا إذا حملت عليه جميع البيوت وانقسمت54 بتلك القسمة البيوت الاربع المتقفة عليها بالحياة والعاقبة والعرس والملك وهي المسمّاة الأوتاد. وأمّا أن نقسم بعضها على وجه ما ثُّ إذا أردنا أن نقسم سائر ها انتقلنا إلى قول

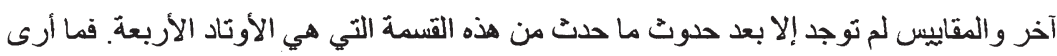
هذا الوجه مرضيا ولا أستحسن سلوكه مؤثر الحقّ ومحيي الحكمة.

\footnotetext{
الأفت Texto, corregido al margen.

${ }^{53}$ En el margen se añade العالم قطب من يعني)

54 انقسمت, repetido en ms.
}

Al-Qanțara (AQ) XXX 1, enero-junio 2009, pp. 41-67 ISSN 0211-3589 
Figura 11

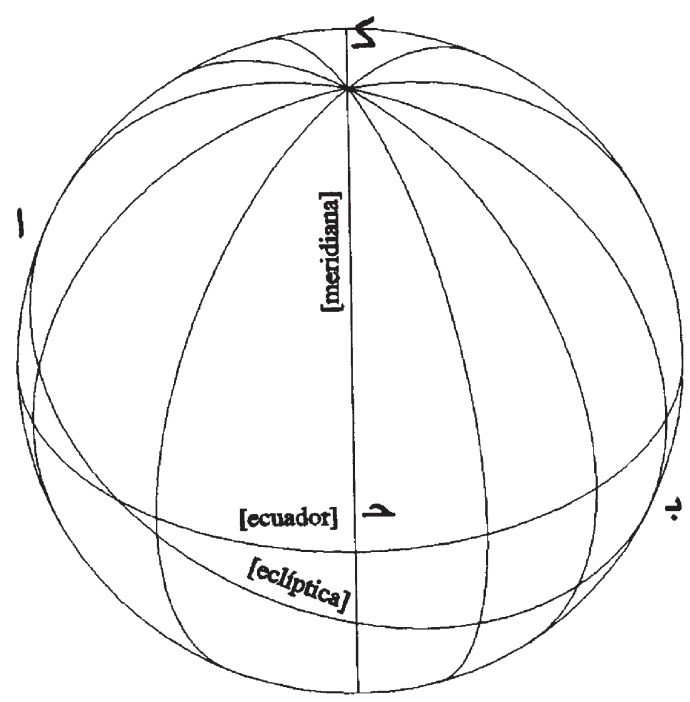

Recibido: $27 / 03 / 2007$

Aceptado: 07/06/2007

Al-Qanțara (AQ) XXX 1, enero-junio 2009, pp. 41-67 ISSN 0211-3589 